\title{
Broncholithiasis Secondary to Pulmonary Actinomycosis
}

\author{
Nicholas R Henry MSc RRT-NPS AE-C and John D Hinze DO
}

\begin{abstract}
We report a case of broncholithiasis secondary to pulmonary actinomycosis. Broncholithiasis has occasionally been reported in association with actinomycosis, but it is unclear if actinomycosis leads to lymph node calcification with subsequent erosion into the airway, producing a broncholith, or if an existing generic broncholith is secondarily colonized with Actinomyces. The patient had postobstruction pneumonia, and computed tomography showed calcified nodules obstructing the bronchus intermedius and distal necrotizing pneumonia. The nodules included sulfur granules containing Gram-positive branching filamentous organisms consistent with Actinomyces. The finding of Actinomyces throughout the broncholith is strong evidence that the etiology of the broncholithiasis was a primary pulmonary Actinomyces infection. Key Words: actinomycosis; Actinomyces; broncholithiasis; broncholith. [Respir Care 2014;59(3):e27-e30. ( 2014 Daedalus Enterprises]
\end{abstract}

\section{Introduction}

Broncholithiasis secondary to pulmonary actinomycosis is a rare condition. ${ }^{1,2}$ Actinomycosis is a chronic pulmonary infection caused by an Actinomyces species, with Actinomyces israelii being the most common pathogen. ${ }^{3-6}$ Actinomyces are Gram-positive bacilli that are not acidfast, and produce branching, filamentous colonies that are round to oval in shape. ${ }^{2,4,5,7}$ Actinomyces colonies produce sulfur granules that are yellowish in color. ${ }^{4,5}$ Actinomyces species are considered normal flora of the human oropharynx, gastrointestinal tract, and urogenital tract,, 5 but can cause infection by invading structures and organs through mucosal lesions. ${ }^{8,9}$ The incidence of actinomycosis is approximately 1 in 300,000 people. ${ }^{9}$ Pulmonary Actinomyces infection is presumed to be caused by aspiration of oropharyngeal secretions, ${ }^{1,5}$ and pulmonary actinomycosis accounts for approximately $15-20 \%$ of actinomycosis cases. ${ }^{8}$ Actinomycosis is confirmed by the isolation and culture of

Mr Henry is affiliated with the Department of Respiratory Care, Texas State University, San Marcos, Texas. Dr Hinze is affiliated with Pulmonary and Critical Care Consultants of Austin, Austin, Texas.

The authors have disclosed no conflicts of interest.

Correspondence: Nicholas R Henry MSc RRT-NPS AE-C, Department of Respiratory Care, Texas State University, 601 University Drive, San Marcos TX 78666. E-mail: NH14@txstate.edu.

DOI: $10.4187 /$ respcare. 02370
Actinomyces colonies and/or the histopathologic finding of sulfur granules..$^{4,7}$

Broncholithiasis is a condition in which calcified nodules or lymph nodes are identified within or migrating into a bronchial lumen. ${ }^{10-12}$ The erosion of the calcified nodule into an airway is a result of continuous movement of the thoracic structures during active breathing or deglutition, ${ }^{5,11,13}$ thereby causing partial or complete obstruction of the airway, potentially leading to an obstructive pneumonia. ${ }^{13}$ Chronic inflammatory processes or granulomatous diseases such as tuberculosis, histoplasmosis, coccidioidomycosis, nocardiosis, cryptococcosis, aspergillosis, and silicosis are common causes of broncholithiasis. ${ }^{1,10-12}$

We describe a case of broncholithiasis secondary to pulmonary actinomycosis. There have been few reports of broncholithiasis coupled with pulmonary actinomycosis, and this case adds useful knowledge and depicts an unusual form of airway obstruction.

\section{Case Report}

A 61-year-old white female presented to the emergency department with weakness, fatigue, increased sputum production, and a 4-month history of hemoptysis. She stated that she smoked tobacco from age 15 to 25 years, with less than 10 pack-years.

Eleven years prior to presentation she developed a chronic productive cough. Eight years later she developed a protracted pneumonia, resulting in substantial weight loss, and was diagnosed with a large pulmonary abscess in 


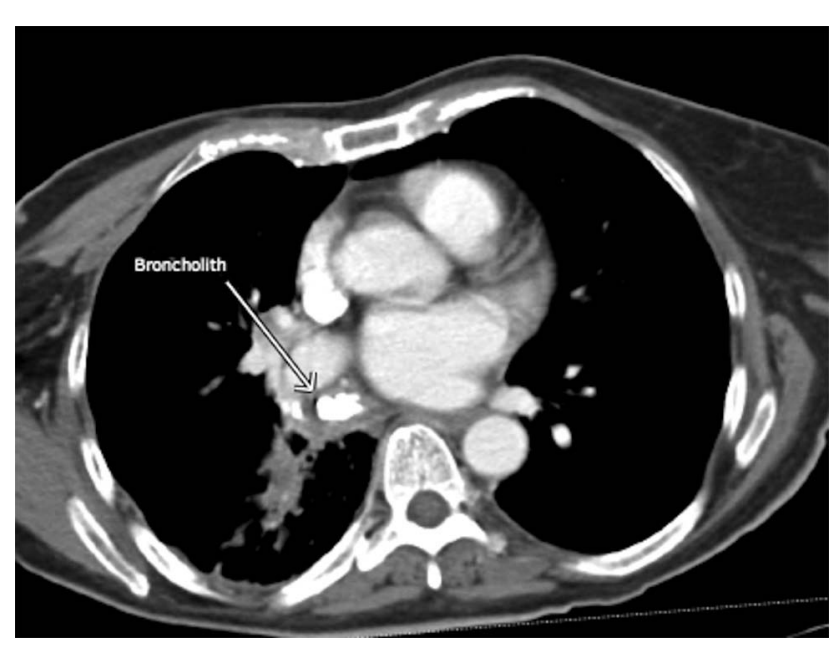

Fig. 1. Computed tomogram shows right hilar lymphadenopathy and a broncholith obstructing the bronchus intermedius.

the right lung, and a percutaneous drain removed approximately $1 \mathrm{~L}$ of fluid. Unfortunately we had no other information regarding the pulmonary abscess, because it was diagnosed and treated at another institution. The patient stated that the abscess fluid cultures were negative for tuberculosis.

Upon presentation to us she appeared chronically ill, with a productive cough that produced yellow sputum with occasional blood streaks. Her highest reported body temperature over the previous 24 hours was $39^{\circ} \mathrm{C}$. Upon presentation her heart rate was 88 beats/min, breathing frequency 24 breaths/min, blood pressure $135 / 62 \mathrm{~mm} \mathrm{Hg}$, and $\mathrm{S}_{\mathrm{pO}_{2}}$ on room air $96 \%$. Her neck had a mildly enlarged anterior cervical lymph node on the right side. She had diminished breath sounds over the right base, and a regular heart rate and rhythm. There was no evidence of digital clubbing, edema, or cyanosis of the extremities. The remainder of her physical exam was unremarkable.

A contrast-enhanced computed tomogram (CT) in the emergency department showed calcified right hilar lymphadenopathy (Fig. 1), near-complete consolidation of the right lower lobe, with cavitation and necrosis, tubular bronchiectasis in the right lower lobe (Fig. 2), an enlarged (short axis $14 \mathrm{~mm}$ ) lymph node anterior to the superior vena cava, and atelectasis and consolidation in the right middle lobe.

She did not have methicillin-resistant Staphylococcus aureus risk factors, but on hospital admission the primary team started her on vancomycin and piperacillin/ tazobactam. During her hospital stay 3 sputum samples were obtained, and all 3 smears were negative for acid-fast Bacillus, and acid-fast Bacillus was not isolated after 6 weeks of culture.

Fiberoptic bronchoscopy found the bronchus intermedius completely filled with creamy purulence. After the

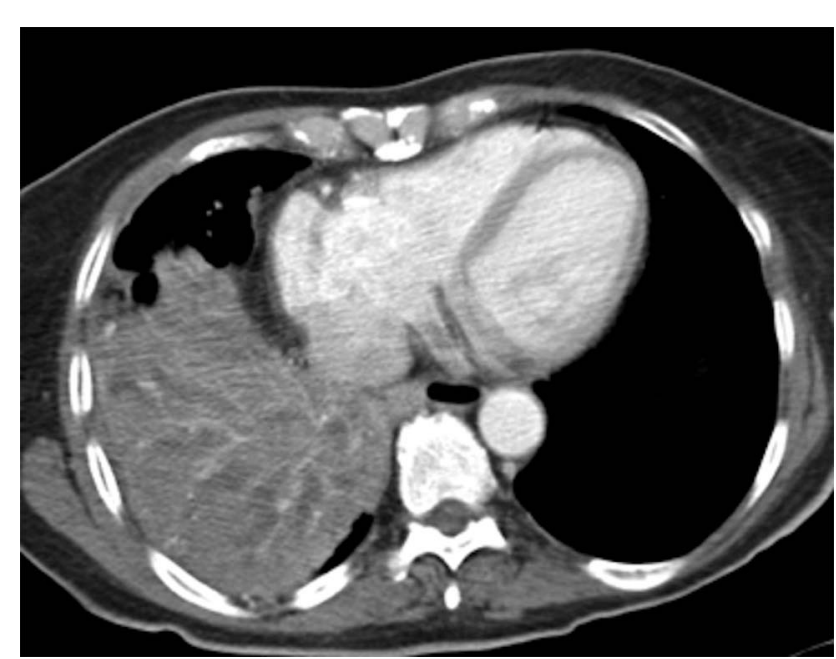

Fig. 2. Computed tomogram shows consolidation and necrosis of the right lower lobe distal to the broncholith.

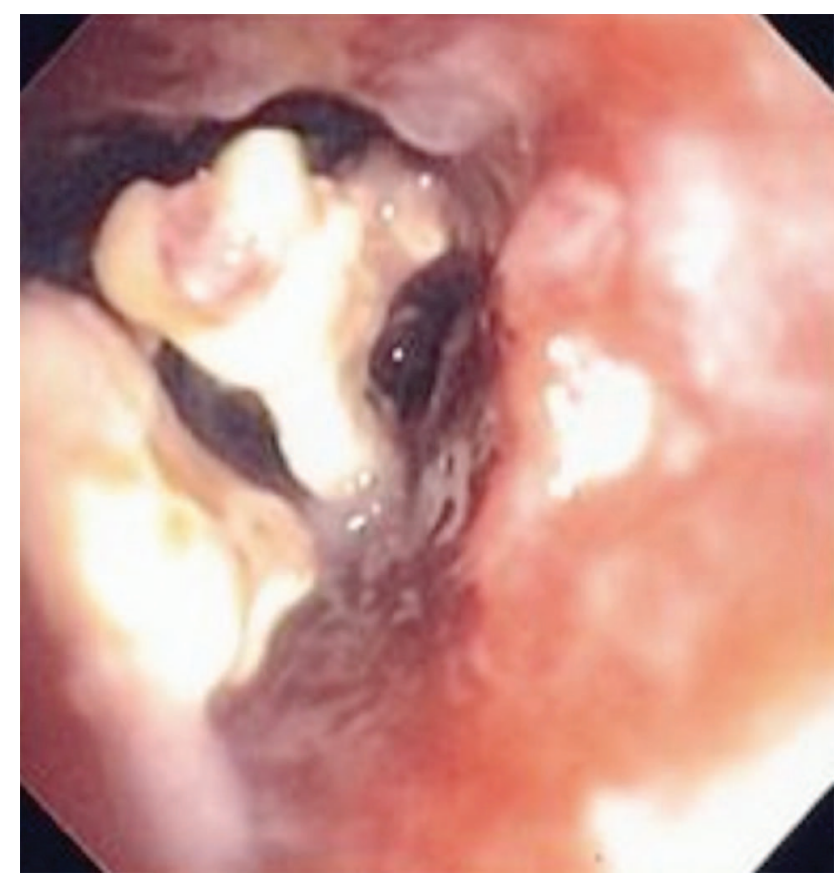

Fig. 3. Bronchoscopy shows 2 large impacted broncholiths occluding the bronchus intermedius.

bronchus intermedius was suctioned clear, several large impacted broncholiths were encountered (Fig. 3). Bronchoalveolar lavage (BAL) of the bronchus intermedius was performed, and 2 small broncholiths were extracted with suction. The BAL had a dense neutrophilic infiltrate with normal respiratory flora, and no anaerobes were isolated after 5 days of culture. The BAL fluid was negative for yeast and fungus via stain and culture, negative for Pneumocystis jirovecii via stain, and negative for acid-fast Bacillus via stain and culture. 


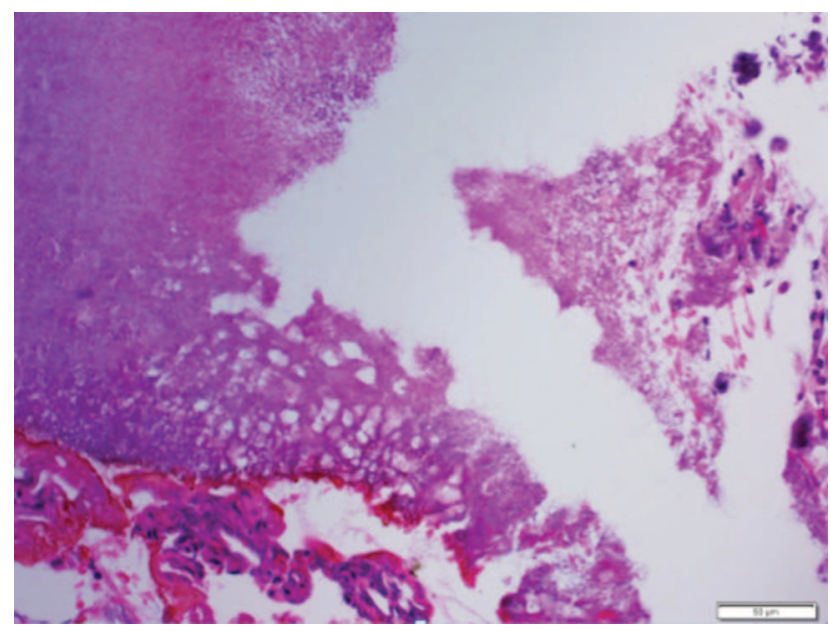

Fig. 4. Microscopy (hematoxylin-eosin stain) of the broncholith shows sulfur granules and branching filaments consistent with Actinomyces.

The remaining broncholiths were extracted in pieces via rigid bronchoscopy, with the assistance of neodymium yttrium aluminum garnet laser ablation. The broncholiths were tan-white, in irregularly shaped fragments ranging from 0.1 to $0.8 \mathrm{~cm}$ in diameter. Microscopy of the broncholiths revealed polypoid fragments of granulation tissue, hemorrhage, calcified material, purulent exudate, actinomycotic sulfur granules, and Gram-positive branching rods throughout the broncholiths (Fig. 4). Gomori methenamine silver stain was negative for fungal organisms. There was no evidence of neoplasm.

The empirical antibiotic treatment was then switched to a continuous infusion of intravenous penicillin. Her symptoms resolved and she was discharged home on a continuous infusion of 12 million units penicillin per day for 4 weeks, followed by oral amoxicillin $(500 \mathrm{mg}, 3$ times a day), which, as ordered, she continued for 4 months, but then stopped taking due to neuropathy. Throughout the out-patient treatment period her symptoms remained abated. A follow-up CT showed modest bronchiectasis in the right lower lobe (Fig. 5) and additional right calcified hilar lymph nodes that had not eroded into an airway (Fig. 6).

\section{Discussion}

Our patient's clinical and radiological findings share many characteristics of previously reported cases of pulmonary actinomycosis: productive cough, hemoptysis, and fever. Other patients have had chest wall pain,2,13 but our patient did not. The radiologic findings from other patients have included air-space consolidation with cavitation, lymphadenopathy, bronchiectasis within the consolidation, localized pleural thickening, and pleural effusion. ${ }^{2,4}$ which, except for pleural thickening and effusion, are also consistent with our patient. Our patient also had CT findings

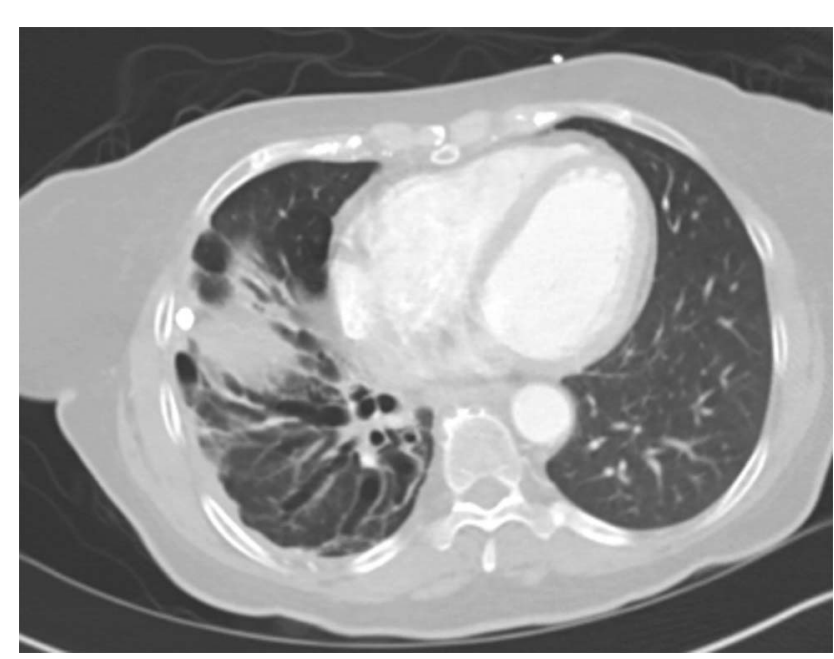

Fig. 5. Computed tomogram shows bronchiectasis in the right lower lobe following antimicrobial treatment.

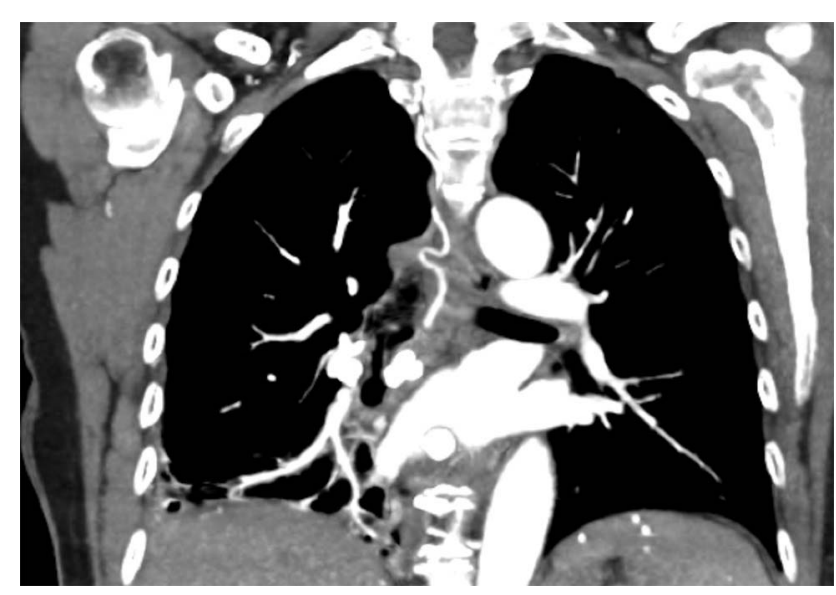

Fig. 6. Coronal reconstruction of a computed tomogram following antimicrobial treatment shows additional right calcified hilar lymph nodes that had not eroded into an airway.

described in other patients with endobronchial actinomycosis associated with broncholithiasis, including a calcified density occluding an airway, distal air-space consolidation, bronchiectasis, and enlarged hilar lymph nodes. ${ }^{1,5}$

In our patient we believe the actinomycosis caused the lymphadenopathy and calcification. Her prolonged course, evidence of pulmonary actinomycosis, and the finding of Gram-positive branching rods and sulfur granules throughout the broncholiths support that the conclusion that the broncholiths were secondary to the infection, and not vice versa. In addition, fungal infection, tuberculosis and bronchogenic carcinoma were ruled out by negative studies. Previous case reports have described endobronchial actinomycosis associated with broncholithiasis, ${ }^{1,5}$ but in our patient sulfur granules were only found within the broncholiths, and Actinomyces was not found in the BAL fluid, so we believe the broncholiths were most likely caused by 


\section{Broncholithiasis Secondary to Pulmonary Actinomycosis}

a parenchymal infection that led to lymphadenopathy, calcification, and erosion into the airway.

Kim et $\mathrm{al}^{5}$ retrospectively reviewed 9 patients with endobronchial actinomycosis in their institution and concluded that in those patients the broncholiths were probably secondarily infected with Actinomyces, which caused the broncholiths to enlarge. We do not believe this occurred in our patient, because there were sulfur granules throughout the broncholiths, rather than only in the periphery of the broncholiths, and we do not think Actinomyces could penetrate an existing broncholith. The fact that the sulfur granules were throughout the broncholiths supports the conclusion that pulmonary actinomycosis can be an etiology of broncholithiasis. The proper management of our patient depended on the definitive and concurrent diagnosis and treatment of both the pulmonary actinomycosis and the broncholithiasis.

\section{ACKNOWLEDGMENTS}

We thank Jordan Weingarten MD, Pulmonary and Critical Care Consultants, Austin, Texas; S Gregory Marshall PhD RRT RPSGT RST, Department of Respiratory Care at Texas State University; and Christopher J Russian MEd RRT-NPS RPSGT RST, Department of Respiratory Care at Texas State University; for reviewing the manuscript.

\section{REFERENCES}

1. Seo JB, Lee JW, Ha SY, Park JW, Jeong SH, Park GY. Primary endobronchial actinomycosis associated with broncholithiasis. Respiration 2003;70(1):110-113.
2. Patel KB, Gupta G, Shah M, Patel P. Pulmonary actinomycosis in fine needle aspiration cytology. J Cytol 2009;26(2):94-96.

3. Hall V. Actinomyces-Gathering evidence of human colonization and infection. Anaerobe 2008;14(1):1-7.

4. Kim TS, Han J, Koh W, Choi JC, Chung MJ, Lee JH, et al. Thoracic actinomycosis: CT features with histopathologic correlation. Am J Roetgenol 2006;186(1):225-231

5. Kim TS, Han J, Koh W, Choi JC, Chung MJ, Lee KS, et al. Endobronchial actinomycosis associated with broncholithiasis: CT findings for nine patients. Am J Roetgenol 2005;185(2):347-353.

6. Clarridge III JE, Zhang Q. Genotypic diversity of clinical Actinomyces species: phenotype, source, and disease correlation among genospecies. J Clin Microbiol 2002;40(9):3442-3448.

7. Reichenbach J, Lopatin U, Mahlaoui N, Beovic B, Siler U, Zbinden $\mathrm{R}$, et al. Actinomycosis in chronic granulomatous disease: an emerging and unanticipated pathogen. Clin Infect Dis 2009;49(11):17031710.

8. Wong VK, Turmezei TD, Weston VC. Actinomycosis. BMJ 2011; 343:d6099.

9. Van Dellen JR. Actinomycosis: an ancient disease difficult to diagnose. World Neurosurg 2010;74(2-3):263-264.

10. Reddy AJ, Govert JA, Sporn TA, Wahidi MM. Broncholith removal using cryotherapy during flexible bronchoscopy: a case report. Chest 2007;132(5):1661-1663.

11. Seo FB, Song K, Lee FS, Goo FM, Kim HY, Song F, et al. Broncholithiasis: review of the causes with radiologic-pathologic correlation. Radiographics 2002;22:S199-S213.

12. Hodgson NC, Inculet RI. Acute airway obstruction secondary to bilateral broncholithiasis. Chest 2000;117(4):1205-1207.

13. Snyder RW, Sawicki RW, Unger M. Bilateral partial bronchial obstruction due to broncholithiasis treated with laser therapy. Chest 1998;113(1):240-242. 